Revue des patrimoines

$24 \mid 2014$

Architecture et urbanisme de villégiature : un état de la recherche

\title{
Les stations thermales : de l'abandon à la renaissance. Une brève histoire du thermalisme en France depuis l'Antiquité
}

\section{Marie-Reine Jazé-Charvolin}

\section{(2) OpenEdition}

Édition électronique

URL : http://journals.openedition.org/insitu/11123

DOI : 10.4000/insitu. 11123

ISSN : 1630-7305

Éditeur

Ministère de la Culture

Référence électronique

Marie-Reine Jazé-Charvolin, « Les stations thermales : de l'abandon à la renaissance. Une brève histoire du thermalisme en France depuis I'Antiquité », In Situ [En ligne], 24 | 2014, mis en ligne le 11 août 2016, consulté le 07 juillet 2020. URL : http://journals.openedition.org/insitu/11123 ; DOI : https://doi.org/10.4000/insitu. 11123

Ce document a été généré automatiquement le 7 juillet 2020

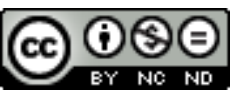

In Situ Revues des patrimoines est mis à disposition selon les termes de la licence Creative Commons Attribution - Pas d'Utilisation Commerciale - Pas de Modification 4.0 International. 
Les stations thermales: de l'abandon à la renaissance. Une brève histoire du thermalisme en France depuis l'Antiquité

Marie-Reine Jazé-Charvolin

(fig. 1) 


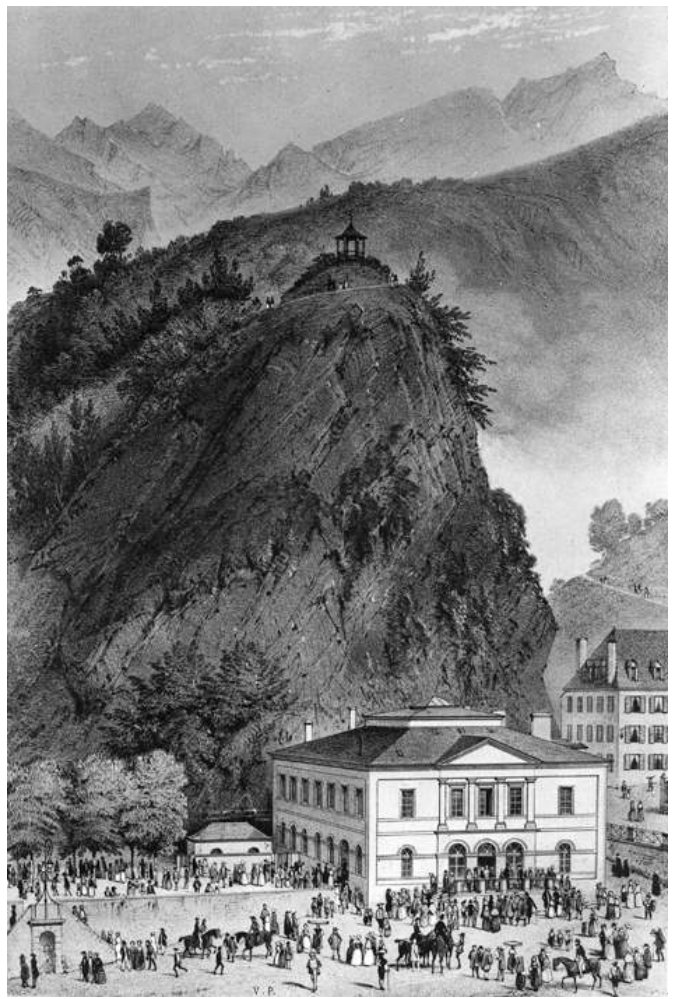

Eaux-Bonnes (Pyrénées-Atlantiques) : établissement thermal et site, dessin et lithographie de Victor Petit, vers 1850.

Repro. Chabot, Bernard ; Dubau, Michel. (c) Région Aquitaine, Inventaire général, 1985.

2 L'existence des stations thermales a pour fondement l'exploitation de sources minérales aux vertus thérapeutiques reconnues. Le mot «thermalisme» est employé pour la première fois en 1845 ; il désigne alors l'usage médical des eaux minérales et ce n'est qu'en 1933 qu'il est utilisé dans son acception actuelle d'exploitation des sources minérales dans des stations thermales. Les villes d'eaux attirent des personnes malades qui viennent chercher là un soulagement à leur souffrance, d'où le lien organique entre thermalisme et médecine, loin de la notion de plaisir associée à la villégiature ${ }^{1}$.

Dès 1605 , Henri IV met en place une véritable organisation du thermalisme. Il crée la Surintendance générale des bains et fontaines du royaume dont il confie la charge à son premier médecin. La surveillance médicale des sources s'organise et ne fera que se renforcer dans les siècles suivants. En 1728, est instituée la Commission pour l'examen des remèdes secrets et des eaux minérales. Par la suite, c'est la Société royale de médecine, fondée en 1778, puis l'Académie royale de médecine, créée en 1820 par Louis XVIII, qui est chargée de tester les qualités curatives des eaux (fig. 2). 


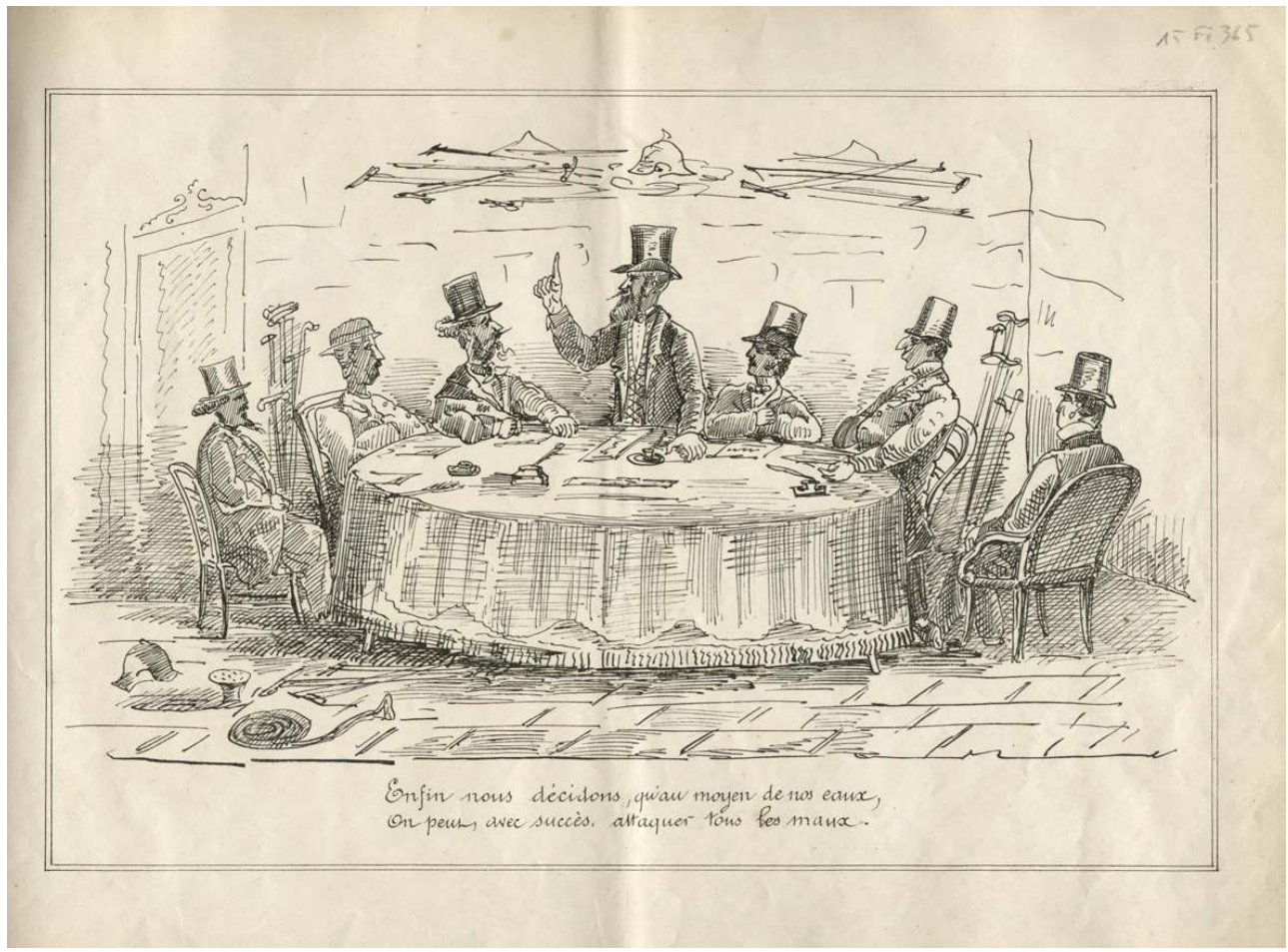

Caricature, dans l'album Une saison d'Eaux à Aix-les-Bains. Dess. Arthur de Varennes de Chinon ; lith. Champod; ed. Perrin, Chambéry, 1868.

(c) Archives municipales d'Aix-les-Bains

Les médecins thermaux eux-mêmes participent de cette démarche. Ils défendent les mérites du traitement mais aussi contribuent à l'évolution des pratiques. En 1832, par exemple, le docteur Constant Despine invente un appareil de douches spéciales qui fait la renommée d'Aix-les-Bains. Le nom de «Berthollet » est donné à cet appareil en hommage à Claude-Louis Berthollet, originaire de la région et diplômé de l'université de médecine de Turin.

\section{Présence du thermalisme sur le territoire}

\section{Répartition}

5 Par le nombre de ses stations thermales, La France est le troisième pays en Europe après l'Allemagne et l'Italie. Elle compte aujourd'hui 108 stations et 112 établissements thermaux habilités par le ministère de la Santé 2 . Toutes se situent globalement à droite d'une ligne sud-ouest/nord-est et, pour $80 \%$ d'entre elles, dans les zones de montagne. Les cinq régions les plus importantes sont l'Aquitaine, le Languedoc-Roussillon, RhôneAlpes, Midi-Pyrénées et l'Auvergne. Les thermes attirent près de 500000 curistes par an, auxquels il faut ajouter environ 300000 accompagnants (fig. 3). 

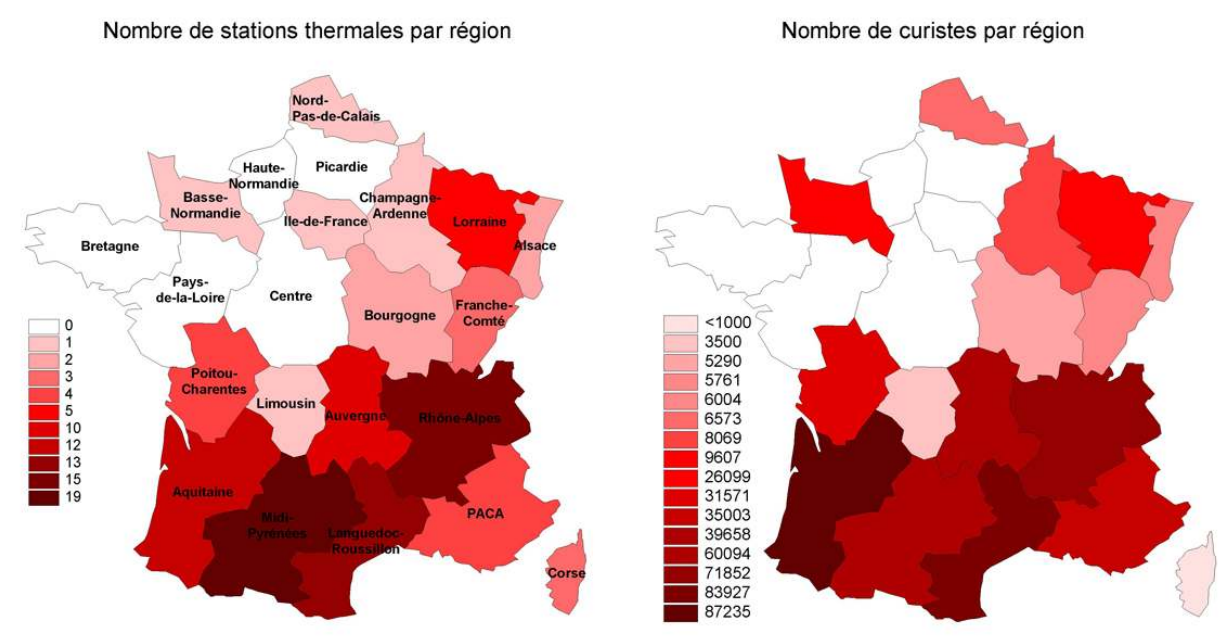

Cartes de répartitions des stations françaises.

Cart. Inv. Cherblanc, P., 2011. (c) Inventaire général, Région Rhône-Alpes.

La première station par sa fréquentation est aujourd'hui Dax, dans les Landes, qui représente à elle seule $10 \%$ de la fréquentation globale. À l'inverse, nombre de petites communes, telle Rennes-les-Bains, dans l'Aude, avec ses 165 habitants, attire moins de 1000 curistes par an. L'orientation thérapeutique d'un établissement est fonction de la composition et de la température de ses eaux, $80 \%$ du total des stations sont spécialisées aujourd'hui dans le traitement des rhumatismes et des affections des voies respiratoires.

\section{Les sources}

$7 \mathrm{Au}$ cours du XIX ${ }^{e}$ siècle, le nombre de sources reconnues pour leurs qualités thérapeutiques explose ${ }^{3}$. La création, en 1854, de la Commission des eaux minérales marque définitivement le passage d'un thermalisme empirique à un thermalisme scientifique. Aujourd'hui, environ 1200 sources sont analysées et surveillées.

8 Cette manne attire les convoitises et incite ses propriétaires à la protéger. Napoléon III, par exemple, décide de l'exclusivité de l'État sur les sources chaudes d'Aix-les-Bains. Parfois, comme à La Bourboule, les possesseurs de sources se livrent à une véritable "guerre des puits» et leurs forages intempestifs conduisent même au tarissement de la ressource ${ }^{4}$.

La découverte des vertus curatives des eaux est souvent fortuite, comme à Marlioz, hameau situé au sud d'Aix-les-Bains, où la tradition veut qu'en 1822, un chimiste espagnol, le chevalier de Gimbernat, ait guéri son acné en se passant sur le visage l'eau qui ruisselait à cet endroit. Mais la population du hameau connaissait déjà les propriétés de cette eau; elle y conduisait les enfants atteints de maladies de peau et y baignait les animaux malades ${ }^{5}$. 


\section{Les établissements thermaux et les différentes étapes de développement}

10 La découverte d'une ou de plusieurs sources détermine l'implantation d'un lieu de cure. L'établissement thermal se doit d'être au plus près du griffon pour ne pas risquer, en multipliant les canalisations, de faire perdre à l'eau ses propriétés et notamment sa température (fig. 4).

Figure 4

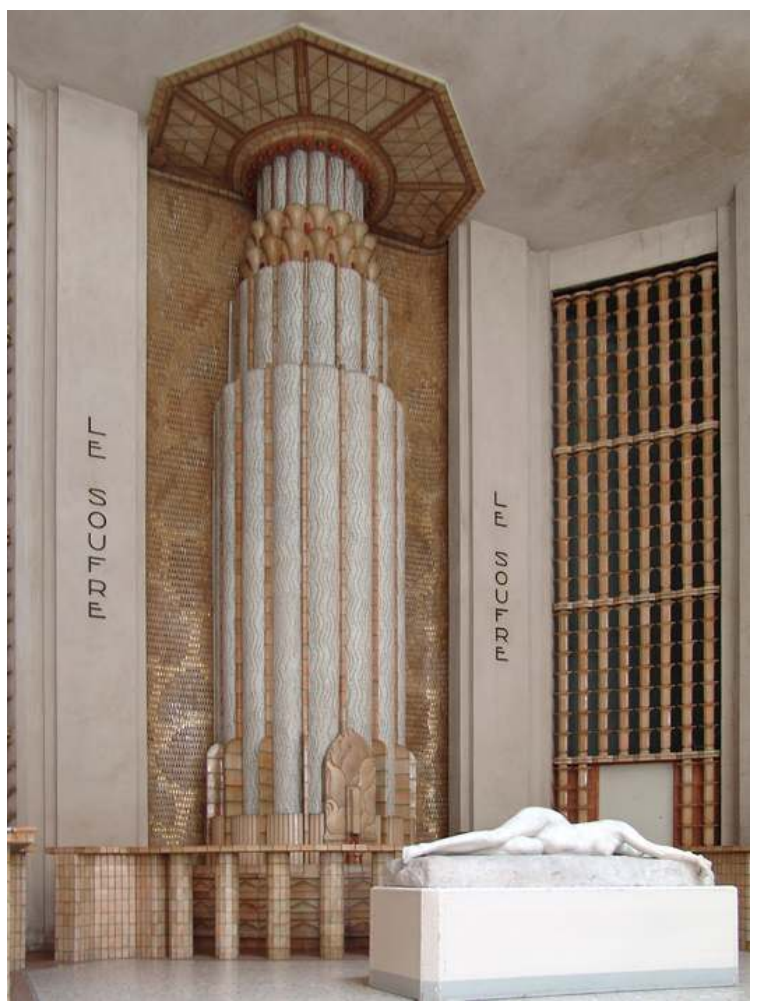

Aix-les-Bains, fontaine de la source de soufre dans le hall des thermes Pétriaux, 1933.

Phot. Inv. Fouger, F. @ Inventaire général, Région Rhône-Alpes/Ville d'Aix-les-Bains.

\section{Les thermes romains}

11 Nombre de stations, telles Néris-les-Bains, Luxeuil-les-Bains, Bourbonne-les-Bains ou Aixles-Bains sont implantées à l'emplacement exact des anciens thermes romains. À l'inverse, la découverte de nouvelles émergences, à Vichy ou Royat notamment, permet de délaisser le site antique. On évalue à $50 \%$ environ les stations actuelles qui conservent des vestiges de thermes romains. Ce passé lointain est encore souvent mis à profit dans les publicités, comme on peut le voir sur le site du centre thermo-ludique de Royatonic. 


\section{Du Moyen Âge au XVII siècle, persistance du thermalisme}

Louis fait édifier à Ax-les-Thermes, en Ariège, un bassin spécial pour soigner les soldats qui ont contracté la peste ; à Plombières-les-Bains, en 1292, un château est construit " pour défendre les baigneurs contre les méchantes gens "; à Bourbonne-les-Bains, du XIV ${ }^{\mathrm{e}}$ au XVII ${ }^{\mathrm{e}}$ siècle, les thermes romains sont cités parmi les revenus des seigneurs ; Bourbonl'Archambault possède les plus célèbres thermes médiévaux et dans la première moitié du xvII ${ }^{e}$ siècle y est édifié le «Logis du Roi » où Talleyrand fait trente et une cures et que fréquentent également $\mathrm{M}^{\mathrm{me}}$ de Sévigné, Boileau, ou encore Fénelon; Rabelais cite les thermes de Néris-les-Bains dans Pantagruel. Au XVII ${ }^{\mathrm{e}}$ siècle, à Aix, les bains ne sont encore que des grottes aménagées et les curistes se font porter l'eau des sources dans leur auberge. La piscine romaine reçoit l'eau de la source d'alun où l'on peut se baigner librement, été comme hiver, dans une eau à $30^{\circ} \mathrm{C}$. Pour des raisons de bienséance, elle sera aménagée en bains des chevaux en 1816. Tous ces exemples montrent une persistance du thermalisme à travers les siècles.

\section{Le XVIIIe siècle, un tournant}

Le mouvement d'amélioration des installations, amorcé au siècle précédent, prend de l'ampleur à partir du milieu du XVIII ${ }^{\mathrm{e}}$ siècle : de véritables établissements thermaux, bien structurés, fonctionnels, avec piscines, séparation des hommes et des femmes, sont mis en chantier. Les constructions se succèdent : Luxeuil en 1762, Plombières en 1771, Aix-enProvence en 1780, Bagnères-de-Bigorre en 1782, Bourbonne-les-Bains et Aix-les-Bains en 1783, Luchon en 1784, Le Mont-Dore en 17857. En 1777, l'Académie d'architecture décide même de consacrer le Grand Prix à un programme complet de thermes.

\section{Le XIXe siècle : la « fièvre thermale ${ }^{8}$}

Sous le Premier Empire déjà, la famille impériale a coutume d'aller prendre les eaux à Bourbonne, Aix ou encore Plombières. Elle donne le ton et entraîne à sa suite un mouvement qui ne cessera qu'un siècle et demi plus tard. La mode des cures thermales se généralise. Quelques nouvelles destinations apparaissent dans les premières décennies, comme Saint-Gervais en 1806, Châtelguyon en 1817, Enghien-les-Bains en 1820, Évian en 1827. Mais c'est autour de 1850 que le thermalisme prend réellement son envol. Dans la deuxième moitié $\mathrm{du} \mathrm{xIx}^{\mathrm{e}}$ siècle, sous l'impulsion notamment de Napoléon III, les anciennes stations s'agrandissent et de nouvelles voient le jour. Des établissements prestigieux, aux programmes architecturaux complexes, aux intérieurs richement décorés, sont édifiés sur tout le territoire. Cette dynamique se poursuit, au $\mathrm{xx}^{\mathrm{e}}$ siècle, jusqu'aux années 1930 environ'.

16 Cependant, selon sa composition et ses propriétés, l'eau thermale n'est pas seulement utilisée en bains et douches dans les piscines et cabines des thermes, mais également en inhalation ou sous forme de boisson, ces diverses indications pouvant bien sûr se 
combiner. En 1850, sur les 200 stations recensées, 75 n'ont pas d'établissement, mais seulement une buvette. L'eau peut également être mise en bouteille sur place et commercialisée, comme à Contrexéville ou Vittel. La réussite d'une ville d'eaux dépend en grande partie de ses investisseurs. Quand, en 1812, Napoléon $\mathrm{I}^{\mathrm{er}}$ achète le domaine thermal de Bourbonne-les-Bains pour le compte de la France, il lui offre une fortune qui ne se démentira plus.

Dans la deuxième moitié du siècle, 7 stations sont gérées par l'État, soit en régie directe comme à Aix-les-Bains, soit indirecte, comme à Vichy ou Plombières. Des communes, regroupements de communes (Cauterets), ou départements (Châtelguyon), possèdent une cinquantaine de stations; ces collectivités pratiquent elles aussi l'affermage (La Bourboule, Le Mont-Dore). Deux cents stations environ sont privées; elles appartiennent à un individu ou une famille, telle la famille Bouloumié qui lance Vittel en 1852, ou à une société capitaliste créée à cet effet, comme à Enghien-les-Bains. Mais les choses peuvent changer. Ainsi, l'État vend Pougues-les-Eaux en 1831 et achète Luxeuil en 1853 ; il cède à nouveau Luxeuil à la ville en $1936^{10}$. Aujourd'hui, l'État ne possède plus aucune station. Il a vendu la dernière, Aix-les-Bains, en 2011, à une société privée, avec l'exclusivité des sources, ce qui interdit toute activité thermale dans les anciens thermes rachetés par la commune.

\section{Vers la villégiature thermale}

Bien sûr, chacune de ces stations ne connaît pas la même prospérité. Une hiérarchie thermale se met ainsi en place. Certaines émergent et deviennent des lieux de villégiature incontournables, tandis que d'autres végètent ou même périclitent. Toutes, cependant, s'adaptent et se transforment pour offrir aux curistes, non seulement les meilleures conditions de soins mais également celles d'un séjour réussi.

\section{Les promenades}

19 Les premiers aménagements vont, dès le $\mathrm{XVIII}^{\mathrm{e}}$ siècle, porter sur la création de promenades, la marche et la "cure de bon air " participant à la réussite du traitement. «Si la promenade et la distraction ne suffisent pas pour guérir, elles y aident sans contredit dans une certaine mesure $\aleph^{11}$. Une allée des Bains est aménagée à Luchon dès 1765, Aix crée la « Promenade du Gigot » en 1783, parallèlement à la construction des thermes royaux. $\mathrm{Au} \mathrm{XIX}^{\mathrm{e}}$ siècle, chaque établissement se doit d'être relié à un parc thermal et cela même si la campagne est proche. Martigny, village des Vosges de moins de 1000 habitants se dote, en 1897, d'un parc paysager de 18 ha et Châteauneuf-les-Bains, petite station de 400 habitants, possède aujourd'hui un parc classé arboretum de 2,5 ha. À Vichy, c'est tout un circuit de promenades couvertes qui est mis en place pour conduire le curiste d'une source à l'autre ${ }^{12}$ (fig. 5). 
Figure 5

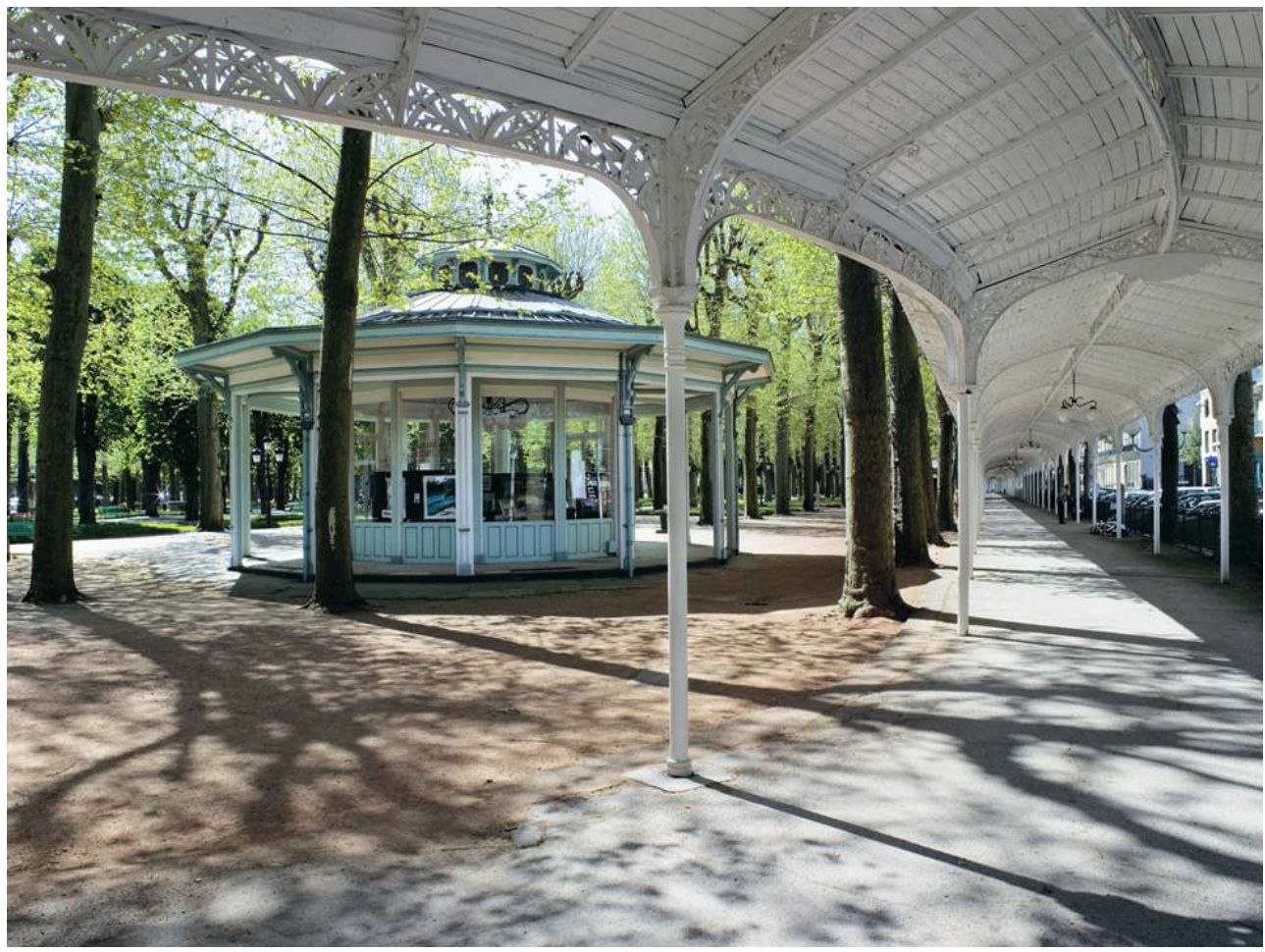

Vichy, pavillon et galerie couverte du Parc des Sources, 1899-1903.

Phot. Inv. Périn, Jean-Michel. (c) Inventaire général, Région Auvergne.

\section{Le logement}

Ces curistes, il faut les loger. Ils descendent dans des auberges, des pensions ou chez l'habitant, tel Lamartine chez le docteur Perrier à Aix. Rares sont encore les véritables hôtels dans la première moitié du XIX ${ }^{\mathrm{e}}$ siècle. Le mouvement s'inverse à partir de 1850 et les hôtels remplacent les pensions. Ces établissements deviennent de plus en plus luxueux pour aboutir, autour de 1900, à la création de palaces dotés du confort le plus moderne ; leur volume est souvent hors d'échelle par rapport à l'urbanisme environnant. Parallèlement à la construction d'hôtels, les habitants aménagent des meublés dans leurs maisons ou immeubles et les « étrangers » se font construire, souvent à la périphérie, de superbes villas (fig. 6). 
Figure 6

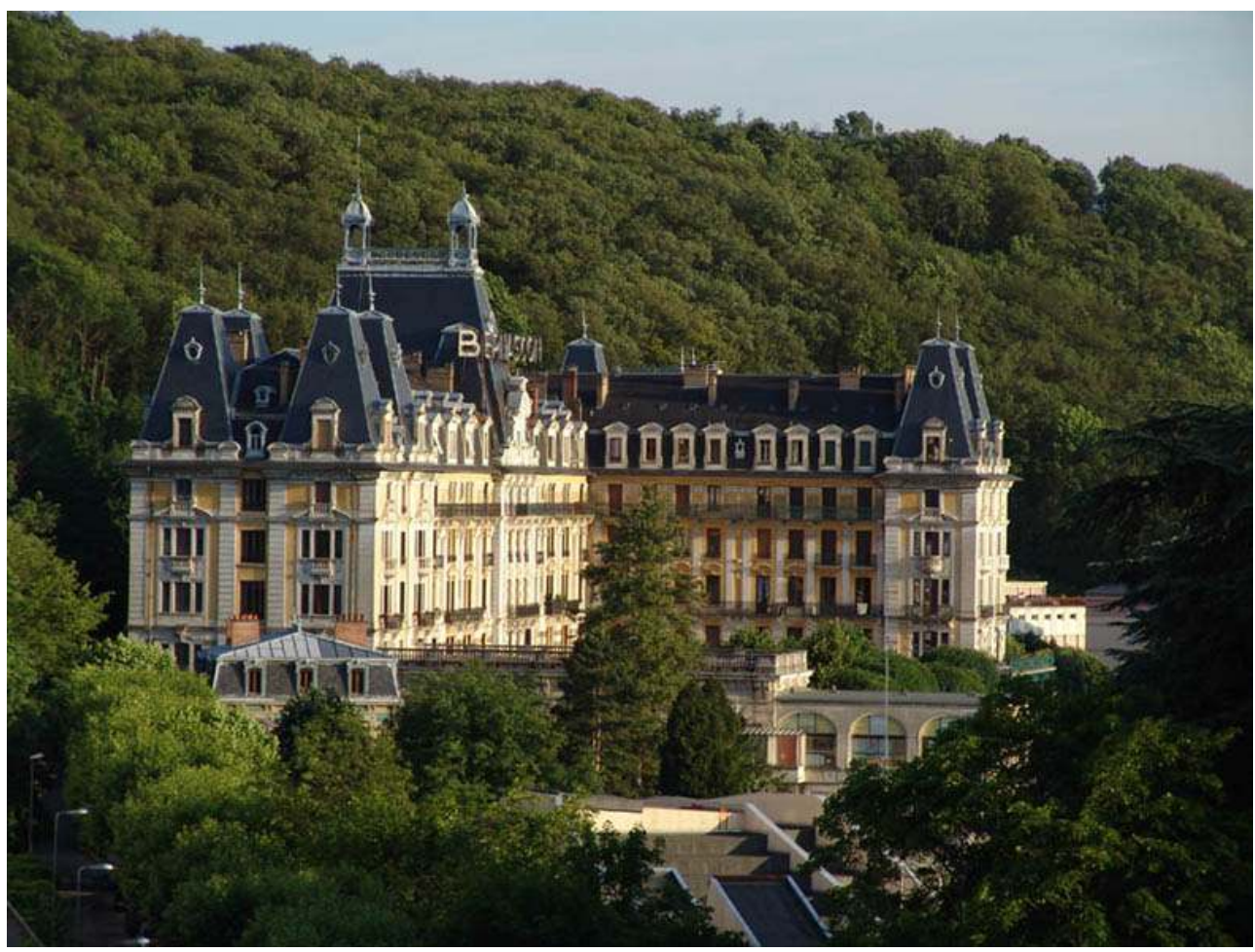

Aix-les-Bains, Hôtel Bernascon, construit entre 1897 et 1900 sur les plans de Jules Pin aîné.

Phot. Inv. Fouger, F. @ Inventaire général, Région Rhône-Alpes/Ville d'Aix-les-Bains.

\section{Les distractions}

En 1806, Napoléon $\mathrm{I}^{\mathrm{er}}$ autorise les jeux de hasard durant la saison thermale. Les cercles, puis les casinos, deviennent des lieux de sociabilité et de vie mondaine qui ne sont pas fréquentés seulement par les curistes. Ainsi, la proximité de Paris pour Enghien-les-Bains, ou de la Suisse pour Évian, constitue-t-elle un atout majeur pour la prospérité du casino et par là même, de la ville. Ces lieux dédiés aux jeux, aux plaisirs et à la rencontre, comportent des salons, une salle de bal et, souvent, un théâtre, à moins que celui-ci ne fasse l'objet d'un programme architectural indépendant. Les stations se dotent également d'équipements sportifs, hippodrome, tennis, golf, plage, offrant ainsi aux villégiateurs un éventail de distractions variées (fig. 7). 
Figure 7

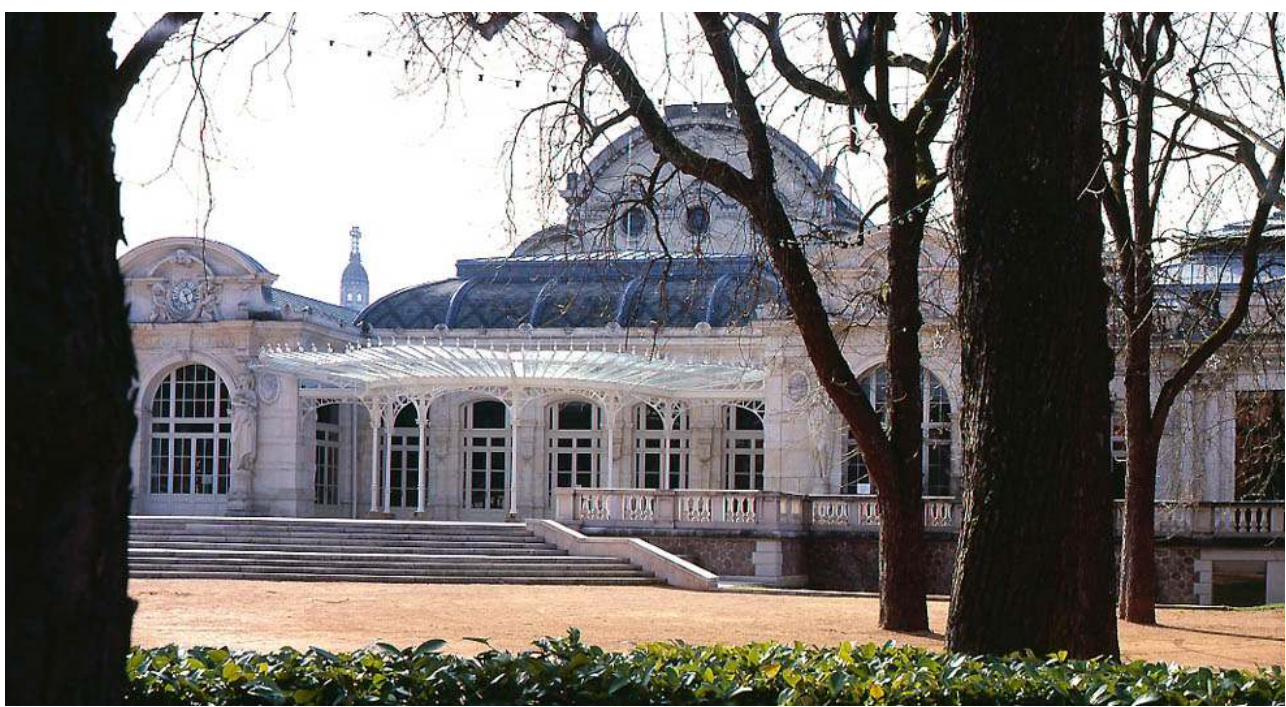

Vichy, grand casino, 1865, agrandi en 1901 par Charles Lecœur et Lucien Woog, aujourd'hui palais des congrès.

Phot. Inv. Périn, Jean-Michel. ( ) Inventaire général, Région Auvergne.

\section{Une fréquentation aux origines multiples}

Tout est ainsi fait pour attirer une clientèle qui va se diversifier et s'élargir, tout au long $\mathrm{du}$ XIX ${ }^{e}$ siècle, dans des proportions variables suivant les stations. Le nombre «d'étrangers» triple entre 1850 et $1870^{13}$. Dans les grandes stations, une clientèle cosmopolite, composée de têtes couronnées, d'aristocrates, de rentiers, d'industriels, d'artistes, afflue chaque été. La liste de ces «étrangers » est publiée dans les gazettes locales, mais il n'est pas toujours facile de faire la distinction entre «baigneurs » ou «curistes» et simple "villégiateur». À Évian, par exemple, ni Anna de Noailles, ni Antoine Lumière, qui se font tous deux construire une villa en bordure du lac, ne semblent avoir fréquenté les thermes. Quoiqu'il en soit, ces visiteurs célèbrent entretiennent la notoriété de la station et attirent de nouveaux clients. Les séjours de Napoléon III à Plombières ou Vichy, ceux de l'impératrice à Eaux-Bonnes et EauxChaudes, dans les Pyrénées, font plus pour le développement de ces stations que la réputation de leurs eaux.

Les étrangers contribuent eux-mêmes souvent à l'aménagement des villes d'eaux : à Aix par exemple, la reine Hortense fonde un hôpital en 1822, Marie de Solms y crée un salon littéraire et construit un théâtre en 1856, et, lors de ses séjours à Aix, la reine Victoria fréquente l'église anglicane fondée par la communauté britannique en 1870. À Contrexéville, l'existence d'une église orthodoxe est due aux visites, durant plus de vingt ans, de la grande duchesse Wladimir de Russie. Celle-ci viendra s'y réfugier en 1917.

\section{Le tournant du thermalisme social}

Après la Seconde Guerre mondiale, une nouvelle clientèle issue du tourisme de masse et du thermalisme social change peu à peu la physionomie des stations. L'aspect médical prend le dessus sur les loisirs. L'image du thermalisme perd de son prestige, notamment 
en raison du vieillissement des clients, les plus de 65 ans représentant $25 \%$ de la fréquentation. Avec le remboursement des cures par la Sécurité sociale, le nombre de curistes augmente, mais, en revanche, la société mondaine déserte les villes d'eaux pour se tourner vers les stations balnéaires ou des destinations plus lointaines. Aujourd'hui, d'après l'IGAS, moins de $2 \%$ d'étrangers sont encore présents dans les stations françaises.

Les orientations d'après-guerre, lourde médicalisation, dépendance de la Sécurité sociale et des pouvoirs publics ${ }^{14}$, changent la donne et c'est tout le patrimoine architectural et paysager qui est touché à partir des années 1960 : les villas de villégiature sont divisées pour faire des meublés ou, comme à La Bourboule ${ }^{15}$, sont transformées en homes d'enfants. Les palaces et grands hôtels, inadaptés à la nouvelle clientèle, sont vendus en copropriété.

À partir de 1993, le thermalisme français connaît une crise qui lui fait perdre 95000 curistes, soit $15 \%$ de sa fréquentation en 7 ans ; en 1996, Aix, la deuxième station française, affiche une perte de $30 \%$ de curistes, et les thermes nationaux sont au bord de la fermeture. Nombre de stations sont en déficit, comme Évaux-les-Bains, en Limousin, ou Luchon, dans les Pyrénées-Atlantiques. Les thermes d'Enghien-les-Bains, près de Paris, en perte de vitesse depuis 1985, ferment en 2002 pour être détruits deux ans plus tard.

\section{Le renouveau}

Face à cette crise, le milieu thermal est amené à se moderniser et à envisager de nouveaux investissements. À la fin du xx $x^{\mathrm{e}}$ siècle, de nombreux établissements (Bourbonne en 1979, Dax en 1982, Monêtier-les-Bains en 1996, Aix en 2000) se substituent aux anciens ou prennent place à côté d'eux. Mais, le plus souvent, ils se repositionnent sur le secteur de la remise en forme ou, du moins, l'intègrent dans leurs offres, en valorisant un thermalisme non conventionné, basé sur la balnéothérapie et le thermo-ludique. L'équilibre est parfois difficile entre image de bien-être et traitements médicaux (fig. 8). 


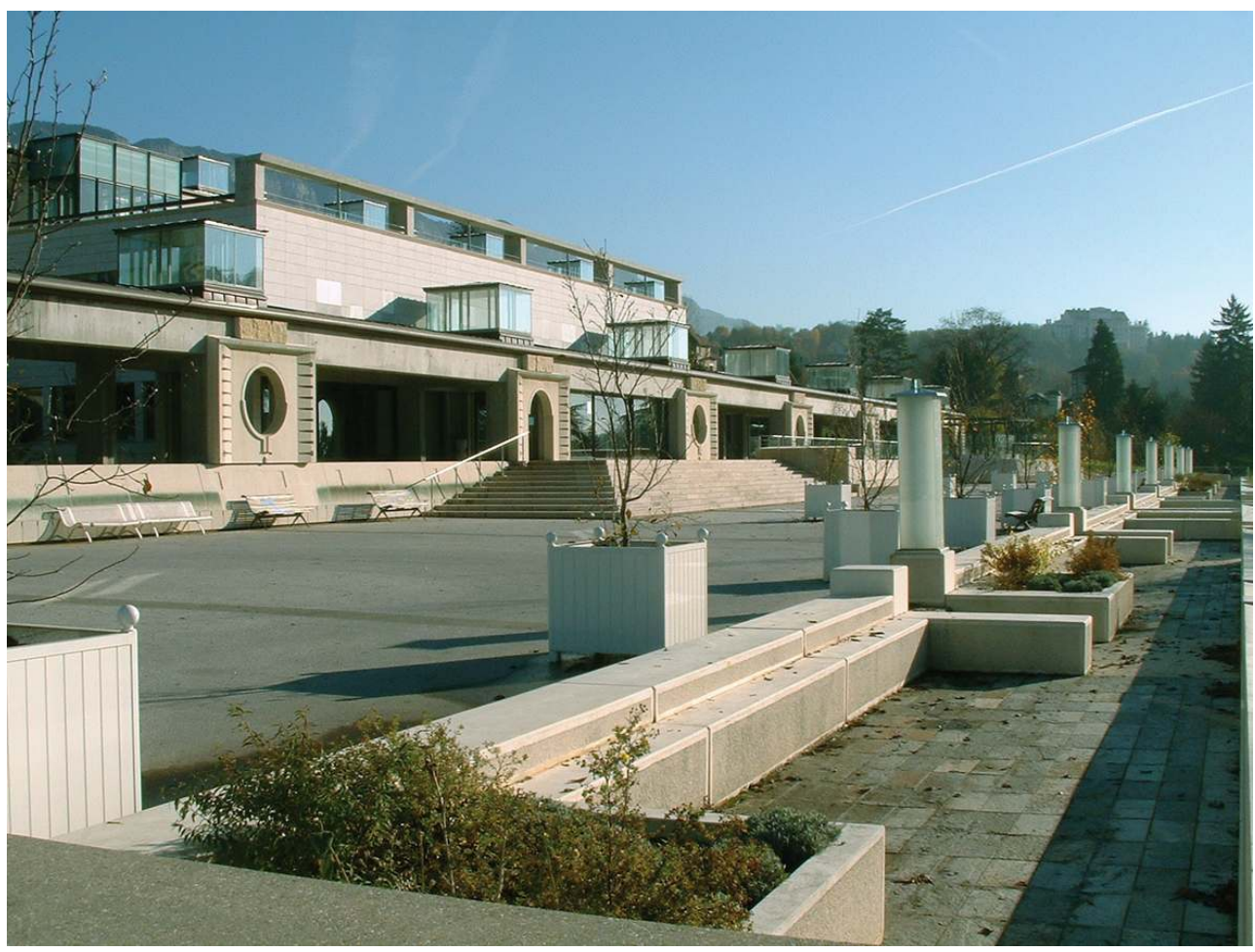

Aix-les-Bains, thermes Chevalley, par Stanislas Fiszer associé à Jean-Yves Mesle, 2000.

Phot. Inv. Fouger, F. ( ) Inventaire général, Région Rhône-Alpes/Ville d'Aix-les-Bains.

D'autres stations mettent l'accent sur le tourisme patrimonial et valorisent leur glorieux passé plus que le thermalisme d'aujourd'hui. En octobre 2008, à Néris-les-Bains, le réseau de la Route des villes d'eaux du Massif central a pris la décision de dresser un inventaire du patrimoine architectural des stations d'Auvergne afin de mieux le faire connaitre. L'ancienne "reine des villes d'eaux ", Vichy, dont la fréquentation a beaucoup baissé, reconvertit ses friches thermales en centre commercial ou en pôle universitaire. De même, à Évian, l'ancien établissement est devenu, en 2006, palais des congrès et centre d'expositions sous la houlette des architectes François Châtillon et Michel Spitz.

Sans réellement abandonner le thermalisme, d'autres stations cherchent à moderniser leur image en jouant la carte du tourisme ; ainsi, depuis plusieurs années, les campagnes promotionnelles d'Aix-les-Bains font appel exclusivement à la présence du lac du Bourget et de la montagne et tournent résolument le dos au passé.

C'est pourquoi, malgré le renouveau, ou à cause de lui, le patrimoine thermal reste aujourd'hui mal connu et menacé. De plus, si la parution, en 1978, de l'article de Bruno Foucart dans le numéro spécial de la revue Monuments historiques, « Retour aux sources ", et la grande exposition organisée en 1984 par l'Institut français d'architecture, intitulée Villes d'eaux en France, avaient suscité de nombreux travaux de recherche sur le sujet, force est de constater qu'aujourd'hui ce thème attire moins. Au sein de l'Inventaire, hormis le service régional de Lorraine qui a un programme d'étude sur plusieurs stations vosgiennes et, bien sûr, l'Auvergne qui vient de publier un ouvrage sur Vichy, peu de chercheurs travaillent sur le thermalisme. 
31 La situation n'est pas meilleure concernant les protections au titre des Monuments historiques. Lors du premier symposium thermal qui s'est tenu à Vichy en 2004, MarieJosé Carroy estimait à $3 \%$ la proportion de patrimoine thermal inscrit ou classé en Auvergne. Pour la France, le pourcentage est encore moindre, puis qu'on ne compte aujourd'hui que 31 établissements thermaux protégés en totalité ou en partie, dont 7 romains, 11 casinos, dont 3 à Vichy, 2 parcs thermaux et 4 buvettes $^{16}$. Puisse ce rapide panorama susciter un renouveau de l'intérêt porté à ce patrimoine exceptionnel.

\section{NOTES}

1. - Ce texte reprend le propos d'une communication faite dans le cadre de la journée d'étude De la villégiature aux lieux de loisirs, tenue le 8 juin 2011 au palais de Chaillot à Paris et organisée par la Cité de l'architecture et du patrimoine à l'initiative du Département du pilotage de la recherche et de la politique scientifique (Direction générale des patrimoines, ministère de la Culture et de la Communication) et avec le concours du Centre André-Chastel. Destinée à servir de socle historique aux débats de la journée d'étude, la communication n'avait pas d'autre ambition que de brosser un rapide panorama d'un phénomène riche et complexe que ces quelques lignes ne peuvent que résumer très sommairement tout en renvoyant aux études monographiques récentes ou en cours.

2. - Chiffres cités dans le rapport de la commission des comptes de la Sécurité sociale de juin 2008, identiques à ceux de la Direction du tourisme de 2004. L'IGAS (Inspection générale des affaires sociales) comptait 107 stations, dont 101 en activité, en 2000. Le Conseil national des exploitants thermaux en recense 105.

3. - 1785 : enquête de l'Académie royale de médecine; $1841,1843,1849,1881,1892$ et 1910 : enquêtes de l'administration des Mines ; 1860 : Dictionnaire des eaux minérales.

4. - CÉRONI, Brigitte, LUNEAU, Jean-François. La Bourboule. Thermalisme et villégiature. Paris : Inventaire général des monuments et des richesses artistiques de la France, 2000, p. 8-9.

5. - MACÉ, $\mathrm{D}^{\mathrm{r}}$, DARDEL, $\mathrm{D}^{\mathrm{r}}$. Le Traitement de Marlioz: eaux sulfureuses, alcalines, iodurées, bromurées, froides. Paris : A. Maloine, 1905, p. 26.

6. - JARRASSÉ, Dominique. Les Thermes romantiques. Bains et villégiature en France de 1800 à 1850. Clermont-Ferrand : Presses universitaires Blaise-Pascal, 1992.

7. - CÉRONI, Brigitte, FIZELLIER-SAUZET, Bernadette. Le Mont-Dore: une ville d'eaux en Auvergne, Puy-de-Dôme. Paris : Inventaire général des monuments et des richesses artistiques de la France, 1998.

8. - PENEZ, Jérôme. Histoire du thermalisme en France au XIX ${ }^{e}$ siècle. Paris : Éditions Economica, 2005, p. 11.

9. - TOULIER, Bernard. Villes d'eaux. Stations thermales et balnéaires. Paris : Imprimerie nationale, 2002, p. 51-69.

10. - GRENIER, Lise (dir.). Villes d'eaux en France. Paris : Institut français d'architecture, 1985, p. 31-32.

11. - FORESTIER, $\mathrm{D}^{\mathrm{r}}$. Promenades d'Aix-les-Bains ou le nouveau guide pratique, médical et pittoresque. 1863, nouvelle éd., p. 59.

12. - RENAULT, Delphine. Vichy ; invitation à la promenade. Lyon : éditions Lieux dits, 2010. 
13. - PENEZ, Jérôme. Histoire du thermalisme en France au XIX ${ }^{e}$ siècle. Paris : Éditions Economica, 2005, p. 28-52.

14. - JARRASSÉ, Dominique. Villes d'eaux des Pyrénées occidentales. Patrimoine et devenir. ClermontFerrand : Presses universitaires Blaise-Pascal, 1996.

15. - CÉRONI, Brigitte, LUNEAU, Jean-François. La Bourboule. Thermalisme et villégiature. Paris : Inventaire général des monuments et des richesses artistiques de la France, 2000, p. 59.

16. - Les 24 restants se répartissant dans 5 régions seulement: 9 dans les Vosges dont 7 à Plombières, 8 en Auvergne, 4 en Midi-Pyrénées, 2 en Rhône-Alpes et 1 en Franche-Comté.

\section{RÉSUMÉS}

Avec 108 stations thermales, la France occupe aujourd'hui le troisième rang en Europe pour le thermalisme. Nombre des établissements thermaux sont implantés à l'emplacement même ou à proximité d'anciens thermes romains. En effet, l'usage des installations antiques a perduré à travers les siècles. Parallèlement, de nouveaux établissements ont vu le jour, notamment à partir $\mathrm{du} \mathrm{XVII}^{\mathrm{e}}$ siècle. Mais c'est autour de 1850 que le thermalisme a pris réellement son envol avec, sur tout le territoire, l'édification d'établissements prestigieux. Bien sûr, chacune des villes d'eaux n'a pas connu la même prospérité. Toutes cependant se sont adaptées et se sont transformées pour offrir aux curistes, non seulement les meilleures conditions de soins mais également celles d'un séjour réussi, se constituant ainsi un patrimoine varié (monuments romains, thermes, casinos, hippodromes, palaces, villas...) doté d'une identité forte et originale. Alors que de nombreux établissements se repositionnent sur le secteur de la remise en forme, l'enjeu d'aujourd'hui est de tirer profit de cette richesse fragile, tout en modernisant les installations pour s'adapter aux nouvelles pratiques.

\section{INDEX}

Mots-clés : baigneur, balnéothérapie, casino, Marie de Solms, palace, parc thermal, piscine, sources, thermalisme, thermes, ville d'eau, villégiature

\section{AUTEUR}

\section{MARIE-REINE JAZÉ-CHARVOLIN}

Ingénieur du Patrimoine reinejaze@orange.fr 